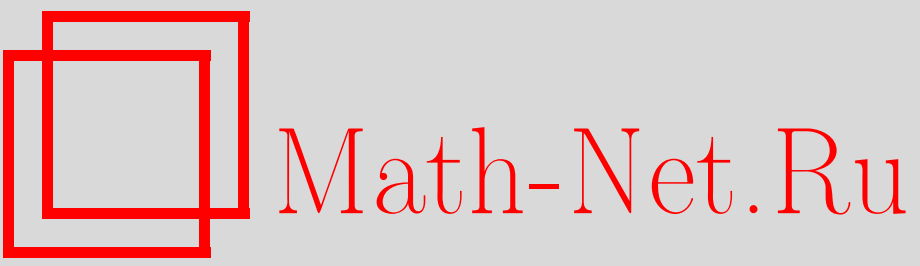

А. Пикеринг, Иерархии Пенлеве и тест Пенлеве, ТМФ, 2003, том 137, номер 3, 445-456

DOI: https://doi.org/10.4213/tmf284

Использование Общероссийского математического портала Math-Net.Ru подразумевает, что вы прочитали и согласны с пользовательским соглашением

http://www.mathnet.ru/rus/agreement

Параметры загрузки:

IP : 18.234 .156 .22

26 апреля 2023 г., 12:53:35 
ТЕОРЕТИЧЕСКАЯ

И МАТЕМАТИЧЕСКАЯ

ФИЗИКА

Том 137, № 3

декабрь, 2003

(C) 2003 г.

А. Пикеринг*

\section{ИЕРАРХИИ ПЕНЛЕВЕ И ТЕСТ ПЕНЛЕВЕ}

В ряде недавних работ мы вывели несколько новых иерархий, являющихся аналогами шести уравнений Пенлеве в высших порядках. В настоящей работе рассмотрен один частный пример такой иерархии, а именно недавно выведенная четвертая иерархия Пенлеве. Она используется для иллюстрации того, как знание гамильтоновых структур и отображений Миуры позволяет найти первые интегралы выведенных обыкновенных дифференциальных уравнений. Рассмотрены также следствия для теста Пенлеве, связанные со вторым членом этой иерархии. В частности, найдено, что алгоритм Абловица-Рамани-Сегура не применим к этому уравнению. Это представляется серьезным недостатком того, что в настоящее время является стандартной проверкой структуры особенностей. Дается решение этой проблемы.

Ключевые слова: тест Пенлеве, иерархии Пенлеве.

\section{1. ВВЕДЕНИЕ}

В последнее время значительный интерес представляет изучение аналогов шести уравнений Пенлеве в высших порядках. Один из подходов состоит в обобшении исходных работ Пенлеве, Гамбье, Шази и др. на классификацию обыкновенных дифференциальных уравнений (ОДУ) высших порядков (см., например, [1]).Другой подход заключается в поиске редукций подобия полностью интегрируемых дифференциальных уравнений в частных производных (ДУЧП) высших порядков; эту идею можно проследить в работе [2], а первые явные результаты приведены в статье [3]. Третий подход, развитый в работах [4]-[7] и являющийся более общим, чем второй, использует связь меж ду неизоспектральными задачами рассеяния и задачами монодромии для ОДУ [8].

В данной работе мы рассмотрим четвертую иерархию Пенлеве, приведенную в статье [7]. Эта иерархия возникает как специальный случай обобщенной $P_{I V}-P_{I I}$ иерархии

$$
\mathcal{R}^{n} \mathbf{u}_{x}+\sum_{i=0}^{n-2} c_{i} \mathcal{R}^{i} \mathbf{u}_{x}+g_{n-1} \mathcal{R}^{2}\left(\begin{array}{l}
1 \\
0
\end{array}\right)+g_{n} \mathcal{R}\left(\begin{array}{l}
1 \\
0
\end{array}\right)+g_{n+1}\left(\begin{array}{l}
1 \\
0
\end{array}\right)=\left(\begin{array}{l}
0 \\
0
\end{array}\right)
$$

* Departamento de Matemáticas, Universidad de Salamanca, Plaza de la Merced, 1, 37008 Salamanca, Spain. E-mail: andrew@gugu.usal.es 
также приведенной в работе [7]. Здесь $\mathbf{u}=(u, v)^{T}, \mathcal{R}$ - рекурсионный оператор иерархии диспергируюших волн на воде (ДВВ) [9] (по поводу дальнейших ссылок см. [7]):

$$
\mathcal{R}=\frac{1}{2}\left(\begin{array}{cc}
\partial_{x} u \partial_{x}^{-1}-\partial_{x} & 2 \\
2 v+v_{x} \partial_{x}^{-1} & u+\partial_{x}
\end{array}\right),
$$

и без потери обшности мы положили $c_{n-1}=0$. Наша четвертая иерархия Пенлеве, определенная в работе [7], соответствует случаю, когда в иерархии (1) $g_{n-1}=0, g_{n} \neq 0$ и все $c_{i}=0$ (однако потоки $\mathcal{R}^{i} \mathbf{u}_{x}$ низшего порядка включаются тривиальным образом).

В разделе 2 показано, как в специальном случае (1) можно получить первые интегралы, используя гамильтонову структуру и отображения Миуры иерархии ДВВ. Тогда для каждого $n$ вместо системы ОДУ порядка $2 n+2$ получается система порядка $2 n$; при $n=1$ получается четвертое уравнение Пенлеве. Эти первые интегралы были приведены в работе [7], но без вывода.

В разделе 3 рассматривается второй член нашей четвертой иерархии Пенлеве. Это уравнение является важным, поскольку оказывается, что алгоритм Абловица-РаманиСегура (AРC) [10] не работает в применении к этому ОДУ, причем ранее неизвестным образом; так называемые резонансы - т.е. положения произвольных коэффициентов в разложении вокруг подвижных особенностей, обычно задаваемые относительно поведения в главном порядке [10], - не могут быть определены. Это представляет собой серьезный недостаток того, что является стандартным тестом структуры особенностей, важным как при тестировании уравнений на интегрируемость (следуя АРC), так и в классификации уравнений со свойством Пенлеве (говорят, что ОДУ обладает свойством Пенлеве, если его общее решение не содержит подвижных особенностей ветвления, при этом особенность определяется как подвижная, если ее положение зависит от констант интегрирования). Мы приводим решение этой задачи, основанное на изучении регулярных особых точек билинейных уравнений и их линеаризаций. Полное описание такого подхода будет дано в работе [11].

Раздел 4 посвяшен выводам и обзору будуших направлений исследований.

\section{2. ИНТЕГРИРОВАНИЕ С ИСПОЛЬЗОВАНИЕМ ГАМИЛЬТОНОВЫХ СТРУКТУР}

В данном разделе показано, как первые интегралы уравнения

$$
\mathcal{R}^{n} \mathbf{u}_{x}+g_{n} \mathcal{R}\left(\begin{array}{l}
1 \\
0
\end{array}\right)+g_{n+1}\left(\begin{array}{l}
1 \\
0
\end{array}\right)=\left(\begin{array}{l}
0 \\
0
\end{array}\right)
$$

можно получить, используя гамильтонову структуру и отображения Миуры иерархии ДВВ. Сначала напомним подробности, касающиеся последней [9]. Иерархия ДВВ является три-гамильтоновой иерархией, хотя мы и будем пользоваться только двумя из ее гамильтоновых операторов $B_{1}$ и $B_{2}$. Они определяются как

$$
\begin{aligned}
B_{1} & =\left(\begin{array}{cc}
0 & \partial_{x} \\
\partial_{x} & 0
\end{array}\right), \\
B_{2} & =\frac{1}{2}\left(\begin{array}{cc}
2 \partial_{x} & \partial_{x} u-\partial_{x}^{2} \\
u \partial_{x}+\partial_{x}^{2} & v \partial_{x}+\partial_{x} v
\end{array}\right) .
\end{aligned}
$$


Рекурсионный оператор $\mathcal{R}$ имеет тогда факторизованный вид $\mathcal{R}=B_{2} B_{1}^{-1}$.

Иерархия ДВВ имеет также отображение Миуры $\mathbf{u}=\mathbf{F}[\mathbf{U}]$, где $\mathbf{U}=(U, V)^{T}$, задаваемое как

$$
\begin{aligned}
& u=U, \\
& v=U V-V^{2}+V_{x} .
\end{aligned}
$$

Гамильтонов оператор $B_{2}$ при этом допускает факторизацию

$$
\left.B_{2}\right|_{\mathbf{u}=\mathbf{F}[\mathbf{U}]}=\mathbf{F}^{\prime}[\mathbf{U}] B\left(\mathbf{F}^{\prime}[\mathbf{U}]\right)^{\dagger},
$$

где $F^{\prime}[\mathbf{U}]$ - производная Фреше отображения Миуры:

$$
\mathbf{F}^{\prime}[\mathbf{U}]=\left(\begin{array}{cc}
1 & 0 \\
V & U-2 V+\partial_{x}
\end{array}\right),
$$

$\left(F^{\prime}[\mathbf{U}]\right)^{\dagger}-$ сопряженное к нему и

$$
B=\frac{1}{2}\left(\begin{array}{cc}
2 \partial_{x} & \partial_{x} \\
\partial_{x} & 0
\end{array}\right)
$$

- гамильтонов оператор соответствующей модифицированной иерархии.

Обратимся теперь к иерархии (3). Определяя последовательность (вариационных производных гамильтоновых плотностей) $\mathbf{L}_{n}[\mathbf{u}]$ как $\mathcal{R}^{n} \mathbf{u}_{x}=B_{2} \mathbf{L}_{n}[\mathbf{u}]=B_{1} \mathbf{L}_{n+1}[\mathbf{u}]$, $\mathbf{L}_{0}[\mathbf{u}]=(0,2)^{T}$, заметим, что (3) можно переписать как

$$
B_{2} \mathbf{L}_{n}[\mathbf{u}]+g_{n} B_{2}\left(\begin{array}{l}
0 \\
x
\end{array}\right)+g_{n+1}\left(\begin{array}{l}
1 \\
0
\end{array}\right)=\left(\begin{array}{l}
0 \\
0
\end{array}\right) .
$$

Начнем с рассмотрения специального случая $g_{n+1}=0$. Сначала используем преобразование Миуры $\mathbf{u}=\mathbf{F}[\mathbf{U}]$, которое приводит к модифицированной иерархии ОДУ

$$
B\left(\mathbf{F}^{\prime}[\mathbf{U}]\right)^{\dagger}\left[\mathbf{L}_{n}[\mathbf{F}[\mathbf{U}]]+g_{n}\left(\begin{array}{l}
0 \\
x
\end{array}\right)\right]=\left(\begin{array}{l}
0 \\
0
\end{array}\right)
$$

которая при заданном виде $B$ приводит к интегрированной модифицированной иерархии

$$
\left(\mathbf{F}^{\prime}[\mathbf{U}]\right)^{\dagger}\left[\mathbf{L}_{n}[\mathbf{F}[\mathbf{U}]]+g_{n}\left(\begin{array}{l}
0 \\
x
\end{array}\right)\right]+\left(\begin{array}{l}
\gamma_{n} \\
\delta_{n}
\end{array}\right)=\left(\begin{array}{l}
0 \\
0
\end{array}\right)
$$

где $\gamma_{n}$ и $\delta_{n}-$ две произвольные постоянные интегрирования.

Рассмотрим теперь замену $\mathbf{F}[\mathbf{U}]$ в последней иерархии на $\mathbf{u}$, что дает соотношения

$$
\begin{gathered}
\mathbf{u}-\mathbf{F}[\mathbf{U}]=\left(\begin{array}{l}
0 \\
0
\end{array}\right) \\
\left(\mathbf{F}^{\prime}[\mathbf{U}]\right)^{\dagger}\left[\mathbf{L}_{n}[\mathbf{u}]+g_{n}\left(\begin{array}{l}
0 \\
x
\end{array}\right)\right]+\left(\begin{array}{l}
\gamma_{n} \\
\delta_{n}
\end{array}\right)=\left(\begin{array}{l}
0 \\
0
\end{array}\right),
\end{gathered}
$$


которые, взятые вместе, определяют преобразование Беклунда между иерархией (13) и соответствуюшей интегрированной версией иерархии (11) при $g_{n+1}=0$. Для получения этой интегрированной иерархии определим

$$
\left(\begin{array}{c}
K_{n} \\
L_{n}
\end{array}\right)=\mathbf{L}_{n}[\mathbf{u}]+g_{n}\left(\begin{array}{l}
0 \\
x
\end{array}\right)
$$

и перепишем (15) в виде

$$
\begin{aligned}
K_{n}+V L_{n}+\gamma_{n} & =0 \\
U L_{n}-2 V L_{n}-L_{n, x}+\delta_{n} & =0 .
\end{aligned}
$$

Затем решим эти уравнения относительно $U$ и $V$ и подставим в (14), что дает

$$
\begin{aligned}
& L_{n, x}=2 K_{n}+u L_{n}+\left(2 \gamma_{n}+\delta_{n}\right), \\
& K_{n, x}=\frac{K_{n}^{2}+\left(2 \gamma_{n}+\delta_{n}\right) K_{n}+\gamma_{n}\left(\gamma_{n}+\delta_{n}\right)}{L_{n}}-v L_{n} .
\end{aligned}
$$

Переопределяя $2 \gamma_{n}+\delta_{n}=g_{n}-2 \alpha_{n}$ и $\delta_{n}^{2}=\beta_{n}^{2}$, получаем

$$
\begin{aligned}
L_{n, x} & =2 K_{n}+u L_{n}+\left(g_{n}-2 \alpha_{n}\right), \\
K_{n, x} & =\frac{\left(K_{n}+g_{n} / 2-\alpha_{n}\right)^{2}-\beta_{n}^{2} / 4}{L_{n}}-v L_{n} .
\end{aligned}
$$

Полученные уравнения являются интегрированными версиями иерархии (11) для случая $g_{n+1}=0$. Это легко проверить, убедившись в том, что $B_{2}\left(K_{n}, L_{n}\right)^{T}=(0,0)^{T}$, когда выполнено (21), (22).

Рассмотрим теперь случай, когда не предполагается, что $g_{n+1}=0$. Переписывая (11) в виде

$$
B_{2} \mathbf{L}_{n}[\mathbf{u}]+\frac{1}{2} g_{n}\left(\begin{array}{c}
(x u)_{x} \\
2 v+x v_{x}
\end{array}\right)+g_{n+1}\left(\begin{array}{l}
1 \\
0
\end{array}\right)=\left(\begin{array}{l}
0 \\
0
\end{array}\right),
$$

видим, что сдвиг $u=w-2\left(g_{n+1} / g_{n}\right)$ при $\mathbf{w}=(w, v)^{T}$ дает

$$
B_{2} \mathbf{L}_{n}[\mathbf{w}]+\sum_{i=0}^{n-1} d_{i} B_{2} \mathbf{L}_{i}[\mathbf{w}]+g_{n} B_{2}\left(\begin{array}{l}
0 \\
x
\end{array}\right)=\left(\begin{array}{l}
0 \\
0
\end{array}\right)
$$

где $d_{i}$ - некоторые постоянные и $B_{2}$ то же, что и ранее, но с заменой $u$ на $w$. Последнее равенство можно записать как

$$
B_{2}\left[\mathbf{L}_{n}[\mathbf{w}]+\sum_{i=0}^{n-1} d_{i} \mathbf{L}_{i}[\mathbf{w}]+g_{n}\left(\begin{array}{l}
0 \\
x
\end{array}\right)\right]=\left(\begin{array}{l}
0 \\
0
\end{array}\right)
$$


и снова рассмотреть модифицированную иерархию, получаемую посредством отображения Миуры $\mathbf{w}=\mathbf{F}[\mathbf{U}]$. Это приводит к интегрированной модифицированной иерархии

$$
\left(\mathbf{F}^{\prime}[\mathbf{U}]\right)^{\dagger}\left[\mathbf{L}_{n}[\mathbf{F}[\mathbf{U}]]+\sum_{i=0}^{n-1} d_{i} \mathbf{L}_{i}[\mathbf{F}[\mathbf{U}]]+g_{n}\left(\begin{array}{l}
0 \\
x
\end{array}\right)\right]+\left(\begin{array}{l}
\gamma_{n} \\
\delta_{n}
\end{array}\right)=\left(\begin{array}{l}
0 \\
0
\end{array}\right),
$$

где $\gamma_{n}$ и $\delta_{n}$ - две произвольные постоянные интегрирования, и, как и выше, к преобразованию Беклунда между последней иерархией и интегрированной версией (24):

$$
\begin{gathered}
\mathbf{w}-\mathbf{F}[\mathbf{U}]=\left(\begin{array}{l}
0 \\
0
\end{array}\right) \\
\left(\mathbf{F}^{\prime}[\mathbf{U}]\right)^{\dagger}\left[\mathbf{L}_{n}[\mathbf{w}]+\sum_{i=0}^{n-1} d_{i} \mathbf{L}_{i}[\mathbf{w}]+g_{n}\left(\begin{array}{l}
0 \\
x
\end{array}\right)\right]+\left(\begin{array}{l}
\gamma_{n} \\
\delta_{n}
\end{array}\right)=\left(\begin{array}{l}
0 \\
0
\end{array}\right) .
\end{gathered}
$$

Записывая это преобразование Беклунда через $\mathbf{u}$ и $\mathbf{U}$, а не через w и $\mathbf{U}$, получаем преобразование Беклунда между иерархией (26) и интегрированной версией (23). Оно имеет вид уравнений

$$
\begin{gathered}
u+2 \frac{g_{n+1}}{g_{n}}=U, \\
v=U V-V^{2}+V_{x},
\end{gathered}
$$

а также

$$
\begin{array}{r}
K_{n}+V L_{n}+\gamma_{n}=0 \\
U L_{n}-2 V L_{n}-L_{n, x}+\delta_{n}=0
\end{array}
$$

где теперь

$$
\left(\begin{array}{c}
K_{n} \\
L_{n}
\end{array}\right)=\mathbf{L}_{n}[\mathbf{u}]+\sum_{i=0}^{n-1}\left(-\frac{g_{n+1}}{g_{n}}\right)^{n-i} \mathbf{L}_{i}[\mathbf{u}]+g_{n}\left(\begin{array}{l}
0 \\
x
\end{array}\right) .
$$

Решение (31), (32) относительно $U$ и $V$ и подстановка в (29), (30) дают теперь в качестве интегрированной формы (23) систему

$$
\begin{aligned}
& L_{n, x}=2 K_{n}+\left(u+2 \frac{g_{n+1}}{g_{n}}\right) L_{n}+\left(g_{n}-2 \alpha_{n}\right), \\
& K_{n, x}=\frac{\left(K_{n}+g_{n} / 2-\alpha_{n}\right)^{2}-\beta_{n}^{2} / 4}{L_{n}}-v L_{n},
\end{aligned}
$$

где $K_{n}$ и $L_{n}$ определяются уравнениями (33), а $\alpha_{n}$ и $\beta_{n}$ снова определены как $2 \gamma_{n}+$ $\delta_{n}=g_{n}-2 \alpha_{n}$ и $\delta_{n}^{2}=\beta_{n}^{2}$. Эту систему можно также представить в эквивалентном виде $\left(I_{n}, J_{n}\right)^{T}=\left(g_{n}-2 \alpha_{n},\left(g_{n} / 2-\alpha_{n}\right)^{2}-\beta_{n}^{2} / 4\right)^{T}$, где

$$
\left(\begin{array}{c}
I_{n} \\
J_{n}
\end{array}\right)=\left(\begin{array}{c}
L_{n, x}-2 K_{n}-\left(u+2 \frac{g_{n+1}}{g_{n}}\right) L_{n} \\
L_{n} K_{n, x}+v L_{n}^{2}+K_{n}^{2}-L_{n, x} K_{n}+\left(u+2 \frac{g_{n+1}}{g_{n}}\right) L_{n} K_{n}
\end{array}\right)
$$

5 Теоретическая и математическая физика, т. 137, № 3, 2003 г. 
Выбор $g_{n+1}=0$ в $(34),(35)$ воспроизводит наш предыдуший результат. Заметим также, что тот же метод можно применить к обобщенному случаю, когда в нашу иерархию включаются потоки младших порядков, т.е. уравнение (1) при $g_{n-1}=0$.

В случае $n=1$ из (34), (35) получаем четвертое уравнение Пенлеве. Таким образом, эти уравнения или, что эквивалентно, уравнение $\left(I_{n}, J_{n}\right)^{T}=\left(g_{n}-2 \alpha_{n},\left(g_{n} / 2-\alpha_{n}\right)^{2}-\right.$ $\left.\beta_{n}^{2} / 4\right)^{T}$, или уравнение (23) действительно составляют четвертую иерархию Пенлеве.

В этом разделе мы проиллюстрировали новый метод получения первых интегралов систем ОДУ. Этот метод, хотя и не сформулированный в работе [7], использовался там для получения первых интегралов (34), (35) (или, эквивалентно, (36)) уравнений (23). Другие случаи, к которым можно применить этот подход, включают, например, обобшенную иерархию ОДУ (3.29) из работы [5].

\section{3. НОВЫЙ МЕТОД НЕЛИНЕЙНОГО АНАЛИЗА ОСОБЕННОСТЕЙ}

Рассмотрим анализ особенностей второго члена нашей четвертой иерархии Пенлеве (34), (35). Полагая $n=2$, получаем [7], [11]

$$
\begin{aligned}
u^{\prime \prime}= & 3 u u^{\prime}-u^{3}-6 u v-2 g_{2} x u-4 g_{3} x+4 \alpha_{2}-8\left(\frac{g_{3}}{g_{2}}\right)^{3}, \\
v^{\prime \prime}= & 2\left(\frac{\left[u v+v^{\prime} / 2-\left(g_{3} / g_{2}\right) v-\alpha_{2}+g_{2} / 2\right]^{2}-\beta_{2}^{2} / 4}{v+u^{2} / 2-u^{\prime} / 2+g_{2} x-\left(g_{3} / g_{2}\right) u+2\left(g_{3} / g_{2}\right)^{2}}\right)- \\
& -2 v\left(v+\frac{1}{2} u^{2}-\frac{1}{2} u^{\prime}+g_{2} x-\frac{g_{3}}{g_{2}} u+2\left(\frac{g_{3}}{g_{2}}\right)^{2}\right)-2(u v)^{\prime}+2 \frac{g_{3}}{g_{2}} v^{\prime},
\end{aligned}
$$

где $\alpha_{2}$ и $\beta_{2}-$ произвольные постоянные.

Решение уравнения (37) относительно $v$ и подстановка в (38) приводят к ОДУ четвертого порядка относительно $u$. Это ОДУ допускает поведение $u \sim u_{0}\left(x-x_{0}\right)^{-1}$ в главном порядке в окрестности подвижной особой точки $x_{0}$ с доминирующим членом

$$
\begin{aligned}
K[u] \equiv & 6 u^{2}\left(u^{\prime \prime}-2 u^{3}\right) u^{\prime \prime \prime \prime}-3 u^{2}\left(u^{\prime \prime \prime}\right)^{2}-6 u u^{\prime}\left(u^{\prime \prime}-8 u^{3}\right) u^{\prime \prime \prime}-8 u\left(u^{\prime \prime}\right)^{3}+ \\
& +9\left(\left(u^{\prime}\right)^{2}-u^{4}\right)\left(u^{\prime \prime}\right)^{2}-12 u^{3}\left(6\left(u^{\prime}\right)^{2}-5 u^{4}\right) u^{\prime \prime}-20 u^{10} .
\end{aligned}
$$

В действительности мы будем рассматривать уравнение $K[u]=0$, поскольку задача определения резонансов связана с доминирующим членом. В частности, мы изучим поведение $u \sim u_{0}\left(x-x_{0}\right)^{-1}$ решения уравнения $K[u]=0$ в главном порядке.

Коэффициент $u_{0}$ в главном порядке (непременно ненулевой) получается из

$$
K\left[u_{0} \xi^{-1}\right]=-20 \xi^{-10} u_{0}^{4}\left(u_{0}^{2}-4\right)\left(u_{0}^{2}-1\right)^{2}=0
$$

где $\xi=x-x_{0}$, что приводит к $u_{0}= \pm 2$ и $u_{0}= \pm 1$ (каждый из этих последних является двойным корнем). Теперь надо найти резонансы, соответствующие каждой из зависимостей $u \sim \xi^{-1}$ и $u \sim 2 \xi^{-1}$ в главном порядке (инвариантность (39) относительно $u \rightarrow-u$ означает, что следует рассматривать только $\left.u_{0}>0\right)$. 
Согласно алгоритму АРС, резонансы определяются подстановкой $u=u_{0} \xi^{-1}+$ $u_{r} \xi^{r-1}$ в доминирующий член и выделением коэффициента линейного по $u_{r}$ члена. Нули получающегося полинома $P\left(r ; u_{0}\right)$ по $r$ являются резонансами; они задают те положения в соответствующем решении в виде ряда, в которых ожидается появление произвольных коэффициентов. Аналогично

$$
P\left(r ; u_{0}\right)=\xi^{10-r} K^{\prime}\left[u_{0} \xi^{-1}\right] \xi^{r-1}
$$

где $K^{\prime}[u]$ - производная $\Phi$ реше от $K[u]$. Таким образом, получаем

$$
P(r ; 2)=\xi^{10-r} K^{\prime}\left[2 \xi^{-1}\right] \xi^{r-1}=-288(r+2)(r+1)(r-4)(r-5),
$$

так что поведение $u \sim 2 \xi^{-1}$ в главном порядке имеет резонансы $r=-2,-1,4,5$. Следовательно, это поведение в главном порядке имеет отрицательные резонансы (помимо резонанса при $r=-1$ ), и его можно исследовать, используя пертурбативньй тест Пенлеве [12], [13].

Однако для поведения $u \sim \xi^{-1}$ в главном порядке получаем

$$
K^{\prime}\left[\xi^{-1}\right] \equiv 0
$$

а потому нельзя найти соответствуюшие резонансы. Таким образом, алгоритм АРС оказывается непригодным для нашего ОДУ. Это ОДУ является первым примером, в котором проявляется такое поведение, хотя очевидно, что он не останется единственным.

Покажем теперь, что можно определить "резонансы” для уравнения $K[u]=0$, соответствующие поведению $u \sim \xi^{-1}$ в главном порядке. В действительности мы найдем, что имеется два множества резонансов, соответствующих данному поведению в главном порядке.

Наше первое замечание относится к появлению двойного корня $u_{0}=1$ (и $u_{0}=-1$ ) уравнения, определяющего коэффициент в главном порядке. Обычно из этого следует, что $r=0$ является резонансом, что далее автоматически приводит к ветвлению [10], [12]. Однако в нашем примере (39) то, что $r=0$ должен быть резонансом, нельзя доказать точно из-за того, что $K^{\prime}\left[\xi^{-1}\right] \equiv 0$.

Очевидно, препятствием при определении резонансов является то обстоятельство, что мы основываемся на линеаризации доминирующих членов (что неявно содержится в алгоритме АРС и становится явным в (41)). Такая линеариация соответствует, разумеется, возмушению вблизи точного решения $u=\xi^{-1}$ уравнения $K[u]=0$ :

$$
K\left[\xi^{-1}+\varepsilon U\right]=K\left[\xi^{-1}\right]+\varepsilon K^{\prime}\left[\xi^{-1}\right] U+\cdots
$$

а потому в данном случае, когда $K^{\prime}\left[\xi^{-1}\right] \equiv 0$, кажется естественным выяснить, какую информацию можно получить из высших порядков по возмушению. Другими словами, введем возмущение

$$
u=\xi^{-1}+\varepsilon U+\varepsilon^{2} V+\varepsilon^{3} W+\varepsilon^{4} X+\cdots,
$$


приводящее к последовательности уравнений (без вкладов в порядках $\varepsilon^{0}$ или $\varepsilon$ )

$$
\begin{array}{ll}
\text { в порядке } \varepsilon^{2}: & F[\xi, U]=0, \\
\text { в порядке } \varepsilon^{3}: & G[\xi, U, V] \equiv F^{\prime}[\xi, U] V+\widetilde{G}[\xi, U]=0, \\
\text { в порядке } \varepsilon^{4}: & H[\xi, U, V, W] \equiv F^{\prime}[\xi, U] W+\widetilde{H}[\xi, U, V]=0,
\end{array}
$$

и подобным же образом в высших порядках по $\varepsilon$, где $F^{\prime}[\xi, U]$ обозначает производную Фреше $F[\xi, U]$ по $U$ (линеаризацию $F[\xi, U]$ вблизи $U$ ).

Здесь уравнение (46), т.е.

$$
\begin{aligned}
F[\xi, U]= & 6 \frac{U^{\prime \prime} U^{\prime \prime \prime \prime}}{\xi^{2}}-36 \frac{U U^{\prime \prime \prime \prime}}{\xi^{4}}-3 \frac{\left(U^{\prime \prime \prime}\right)^{2}}{\xi^{2}}+6 \frac{U^{\prime \prime} U^{\prime \prime \prime}}{\xi^{3}}+36 \frac{U^{\prime} U^{\prime \prime \prime}}{\xi^{4}}-108 \frac{U U^{\prime \prime \prime}}{\xi^{5}}- \\
& -48 \frac{\left(U^{\prime \prime}\right)^{2}}{\xi^{4}}+108 \frac{U^{\prime} U^{\prime \prime}}{\xi^{5}}+216 \frac{U U^{\prime \prime}}{\xi^{6}}-108 \frac{\left(U^{\prime}\right)^{2}}{\xi^{6}}-216 \frac{U U^{\prime}}{\xi^{7}}=0
\end{aligned}
$$

является (с необходимостью) билинейным уравнением по $U$ и может быть получено как

$$
F[\xi, U]=\left.\frac{1}{2}\left(\frac{d^{2}}{d \varepsilon^{2}} K\left[\xi^{-1}+\varepsilon U\right]\right)\right|_{\varepsilon=0}=\frac{1}{2} \sum_{i=0}^{4} \sum_{j=0}^{4} \frac{\partial^{2} K}{\partial u^{(i)} \partial u^{(j)}}\left[\xi^{-1}\right] U^{(i)} U^{(j)}=0 .
$$

Более того, по аналогии с линейными уравнениями понятно, что $\xi=0$ можно определить как регулярную особую точку этого билинейного уравнения; каждый коэффицциент при $U^{(i)} U^{(j)}$ в уравнении (49) является аналитичным при $\xi=0$ после умножения на $\xi^{8-i-j}$. Таким образом, подстановка $U=\xi^{\alpha}$ приводит к "indicial" уравнению

$$
(\alpha+2)^{2} \alpha(\alpha-3)^{2}(\alpha-4)=0 .
$$

Поиск решения в виде ряда, начиная с младшего корня indicial уравнения, приводит нас к обшему решению уравнения (49)

$$
U=U_{-1} \xi^{-2}+U_{1}+U_{3} \xi^{2}+U_{4} \xi^{3}+U_{5} \xi^{4},
$$

где $U_{1}, U_{3}$ и $U_{5}$ подчинены связи ${ }^{1)}$

$$
U_{3}^{2}+9 U_{1} U_{5}=0
$$

Уместны несколько замечаний. Во-первых, indicial уравнение имеет шесть корней; это так, потому что (49) является билинейным и включает члены, полное число производных в которых равно шести. В отличие от случая линейных уравнений, повторяюшиеся корни не приводят к логарифмическим членам. Во-вторых, корни indicial уравнения дают степени $\xi$, которые являются решениями уравнения; однако - снова в отличие от

\footnotetext{
1) Без этой связи решение (52) является общим решением линейного ОДУ $\xi^{5} U^{\prime \prime \prime \prime \prime}+3 \xi^{4} U^{\prime \prime \prime \prime}-$ $9 \xi^{3} U^{\prime \prime \prime}+18 \xi^{2} U^{\prime \prime}-18 \xi U^{\prime}=0$. Однако, вводя возмущение, мы получаем именно (49).
} 
обычного линейного анализа - они не соответствуют (главным) степеням $\xi$ в решении в виде ряда, чьи коэффициенты остаются произвольными. В действительности в решении (52) имеется произвол относительно того, какие из пяти коэффициентов следует рассматривать как произвольные. Как мы увидим ниже, ответ на этот вопрос основан на решениях уравнений, возникающих в высших порядках по $\varepsilon$. В третьих, мы пронумеровали коэффициенты разложения для $U$ относительно доминируюшего поведения $u$, т.е. $u=\xi^{-1}+\varepsilon \xi^{-1}\left[U_{-1} \xi^{-1}+U_{1} \xi+U_{3} \xi^{3}+U_{4} \xi^{4}+U_{5} \xi^{5}\right]+O\left(\varepsilon^{2}\right)$.

Именно уравнение (49) составляет основу нашего подхода к пониманию структуры особенностей уравнения $K[u]=0$ (где $K[u]$ определено в $(39))$. Этот подход, с помощюю которого мы изучаем регулярные особые точки билинейного, а не линейного уравнения (см. (41)) с целью найти резонансы, является новым. Однако для анализа информации, полученной из ОДУ (49), и для выяснения того, какие из коэффициентов в (52) соответствуют резонансам, нам требуются высшие порядки по возмушению.

В третьем порядке по $\varepsilon$ получаем неоднородное линейное уравнение для $V$ с коэффициентами при линейных членах, зависящими от $U_{1}, U_{3}$ и $U_{5}$. Рассмотрим сначала случай $U_{1} \neq 0$; тогда в нашем уравнении доминируюшая линейная часть по $V$ имеет вид

$$
-\frac{36}{\xi^{4}} U_{1}\left(V^{\prime \prime \prime \prime}+\frac{3}{\xi} V^{\prime \prime \prime}-\frac{6}{\xi^{2}} V^{\prime \prime}+\frac{6}{\xi^{3}} V^{\prime}\right) .
$$

Для этой доминирующей линейной части соответствующее indicial уравнение с нумерацией, снова заданной относительно поведения $u$ в главном порядке, определяется как

$$
\xi^{5-r}\left(\frac{d^{4}}{d x^{4}}+\frac{3}{\xi} \frac{d^{3}}{d x^{3}}-\frac{6}{\xi^{2}} \frac{d^{2}}{d x^{2}}+\frac{6}{\xi^{3}} \frac{d}{d x}\right) \xi^{r-1}=(r+1)(r-1)(r-3)(r-4)=0,
$$

и таким образом в решении для $V$ в виде ряда коэффициенты $V_{-1}, V_{1}, V_{3}$ и $V_{4}$ (соответствуюшие $\xi^{-2}, \xi^{0}, \xi^{2}$ и $\left.\xi^{3}\right)$ будут произвольными. Неоднородные члены определяют поведение $V$ в главном порядке как $V \sim U_{-1}^{2} \xi^{-3}$, и, таким образом, в данном случае $U_{1} \neq 0$ мы получаем обшее решение нашего уравнения для $V$ в виде

$$
\begin{aligned}
V= & \xi^{-1}\left[U_{-1}^{2} \xi^{-2}+V_{-1} \xi^{-1}+V_{1} \xi-\left(U_{1}^{2}+2 U_{-1} U_{3}\right) \xi^{2}+V_{3} \xi^{3}+V_{4} \xi^{4}+\right. \\
& +\left(\frac{5}{8} U_{1} U_{4}-\frac{2}{3} \frac{U_{-1} U_{3} U_{4}}{U_{1}}-\frac{U_{5}}{U_{1}} V_{1}-\frac{2}{9} \frac{U_{3}}{U_{1}} V_{3}\right) \xi^{5}+\frac{3}{7} U_{1} U_{5} \xi^{6}+ \\
& \left.+\frac{7}{24} U_{3} U_{4} \xi^{7}+\left(\frac{1}{6} U_{4}^{2}+\frac{8}{21} U_{3} U_{5}\right) \xi^{8}+\frac{3}{8} U_{4} U_{5} \xi^{9}+\frac{15}{77} U_{5}^{2} \xi^{10}\right],
\end{aligned}
$$

где $U_{1}, U_{3}$ и $U_{5}$ снова подчинены (53), а $V_{-1}, V_{1}, V_{3}$ и $V_{4}$ остаются произвольными при том, что выполнены условия совместности.

Теперь мы в состоянии интерпретировать наши результаты в рассматриваемом случае $U_{1} \neq 0$. Прежде всего заметим, что в этом случае естественная интерпретация (52) состоит в том, что коэффициенты $U_{-1}, U_{1}, U_{3}$ и $U_{4}$ произвольны, и что $U_{5}$ определяется как $U_{5}=-U_{3}^{2} /\left(9 U_{1}\right)$. Фиксируя теперь произвольные коэффициенты в нашем пертурбативном разложении (45), видим, что в каждом порядке $\geqslant 1$ по возмушению коэффициенты при $\xi^{r-1}$ при $r=-1,1,3,4$ произвольны (напомним, что все линейные части 
уравнений, получаемых в порядках $\geqslant 3$ по возмушению, одни и те же). Эти произвольные коэффициенты, занумерованные относительно поведения $u \sim \xi^{-1}$ в главном порядке, являются в точности искомыми резонансами.

Другими словами, мы получили, что соответственно поведению $u \sim \xi^{-1}$ в главном порядке мы имеем резонансы $r=-1,1,3,4$. Таким образом, видно, что можно найти резонансы для поведений в главном порядке, подобных исследуемому здесь, несмотря на то что выполнено уравнение, соответствуюшее (43). Это в свою очередь означает, что мы в состоянии наложить условие целочисленности любых резонансов, определенных указанным образом и даюших информацию об общем решении уравнения, если предполагается, что уравнение обладает свойством Пенлеве. Другими словами, можно обобшить обычное условие целочисленности резонансов [12], [13] на системы типа рассматриваемой в данной работе. (То же условие можно наложить на корни indicial уравнения для соответствуюшего билинейного уравнения, относительно которого снова известно, что его корни дают информацию об обшем решении исходного уравнения.)

Рассмотрим теперь случай $U_{1}=0$; из $(53) U_{3}=0$, и наше уравнение для $V$ принимает вид

$$
\begin{aligned}
& 36 U_{5}\left(V^{\prime \prime \prime \prime}-\frac{1}{\xi} V^{\prime \prime \prime}-\frac{6}{\xi^{2}} V^{\prime \prime}+\frac{30}{\xi^{3}} V^{\prime}-\frac{48}{\xi^{4}} V\right)- \\
& \quad-\frac{7560}{\xi^{7}} U_{-1}^{2} U_{5}-3240 \xi^{3} U_{4}^{2} U_{5}-16200 \xi^{4} U_{4} U_{5}^{2}-16200 \xi^{5} U_{5}^{3}=0
\end{aligned}
$$

Предположим, что $U_{5} \neq 0$; соответствующее indicial уравнение для (57), вновь с нумерацией относительно поведения $u$ в главном порядке, имеет вид

$$
\xi^{5-r}\left(\frac{d^{4}}{d x^{4}}-\frac{1}{\xi} \frac{d^{3}}{d x^{3}}-\frac{6}{\xi^{2}} \frac{d^{2}}{d x^{2}}+\frac{30}{\xi^{3}} \frac{d}{d x}-\frac{48}{\xi^{4}}\right) \xi^{r-1}=(r+1)(r-3)(r-4)(r-5)=0
$$

так что в решении уравнения (57) в виде ряда коэффициенты $V_{-1}, V_{3}, V_{4}$ и $V_{5}$ (соответственно при $\xi^{-2}, \xi^{2}, \xi^{3}$ и $\left.\xi^{4}\right)$ будут произвольными. Поведение в главном порядке определяется членом при $\xi^{-7}$ как $V \sim U_{-1}^{2} \xi^{-3}$, и (57) имеет общее решение

$$
V=\xi^{-1}\left[\frac{U_{-1}^{2}}{\xi^{2}}+\frac{V_{-1}}{\xi}+V_{3} \xi^{3}+V_{4} \xi^{4}+V_{5} \xi^{5}+\frac{U_{4}^{2}}{6} \xi^{8}+\frac{3}{8} U_{4} U_{5} \xi^{9}+\frac{15}{77} U_{5}^{2} \xi^{10}\right],
$$

где $V_{-1}, V_{3}, V_{4}$ и $V_{5}$ остаются произвольными при том, что выполнены условия совместности.

Обратимся теперь к интерпретации наших результатов в рассматриваемом случае $U_{1}=U_{3}=0$ и $U_{5} \neq 0$. Мы видим, что в первом порядке по $\varepsilon$ имеются три произвольных коэффициента, $U_{-1}, U_{4}$ и $U_{5}$; во всех последующих порядках по $\varepsilon$ имеем четыре произвольных коэффициента, а именно коэффициенты при $\xi^{r-1}, r=-1,3,4,5$ (например, $V_{-1}, V_{3}, V_{4}$ и $V_{5}$ во втором порядке по $\varepsilon$ ). Эти произвольные коэффициенты, занумерованные относительно поведения $u$ в главном порядке, образуют отдельный набор резонансов, соответствуюших поведению $u \sim \xi^{-1}$ в главном порядке. Это дает первый 
пример уравнения, где для поведения в главном порядке найдены два различных множества резонансов.

В описанном выше способе нахож дения резонансов, соответствуюших поведению $u \sim$ $\xi^{-1}$ в главном порядке, заметим, что по крайней мере во втором случае $\left(U_{1}=U_{3}=0\right.$, $\left.U_{5} \neq 0\right)$, решаюшую роль играет линеаризация нашего билинейного уравнения. В нашем решении для $U$ в этом случае имеем три произвольных коэффициента $\left(U_{-1}, U_{4}\right.$ и $\left.U_{5}\right)$, и четвертый резонанс получается только из нашего линейного уравнения для $V$. Это важно при рассмотрении решений в виде ряда.

Рассмотрим конструкцию решений уравнения $K[u]=0$ в виде ряда, когда $K[u]$ имеет вид (39), для поведения $u \sim u_{0} \xi^{-1}$ в главном порядке, или другими словами, конструкцию решений в виде ряда (разложений Пенлеве)

$$
u=\xi^{-1} \sum_{j=0}^{\infty} u_{j} \xi^{j},
$$

где все $u_{j}$ являются постоянными. В случае $u_{0}=2$ можно, как обычно, построить разложение Пенлеве и найти, что, пока мы имеем дело с резонансами $r=-2,-1,4,5$, коэффициенты $u_{4}$ и $u_{5}$ остаются произвольными при выполненни условий совместности. Как отмечалось выше, более полно это поведение в главном порядке можно рассмотреть, используя пертурбативный тест Пенлеве [12], [13].

Однако случай $u_{0}=1$ приводит к весьма необычному поведению. Поскольку $K^{\prime}\left[\xi^{-1}\right] \equiv 0$, коэффициенты $u_{j}$ определяются уже не рядом линейных алгебраических уравнений, а рядом нелинейных алгебраических уравнений. Таким образом, определение коэффициентов $u_{j}$ из (60) разбивается на ряд подслучаев.

Если мы предполагаем, что $u_{1} \neq 0$, то получаем решение $(60)$, в котором $u_{1}, u_{3}$ и $u_{4}$ произвольны, а остальные $u_{j}$, по всей видимости, определены в терминах трех указанных. Это, очевидно, соответствует первому случаю, приведенному выше (резонансы $r=-1,1,3,4)$.

При $u_{1}=0$ имеем $u_{2}=u_{3}=0$, и $u_{4}$ и $u_{5}$ остаются произвольными (если $u_{5} \neq 0$ ), а остальные $u_{j}$, как кажется, определяются в терминах этих двух. Это соответствует второму случаю, приведенному выше, причем $r=3$ ведет себя как отрицательный резонанс (т.е. ему можно присвоить произвольные данные только с помощью пертурбативного разложения).

Последний случай указывает, что использование одной только конструкции (60) для исследования поведения решений уравнения $K[u]=0$ в главном порядке $u \sim \xi^{-1}$ привело бы к ошибкам. Без определения соответствуюших полных наборов резонансов нельзя исключить рациональное ветвление.

\section{4. ЗАКЛЮЧЕНИЕ}

В данной работе мы рассмотрели четвертую иерархию Пенлеве, приведенную в статье [7]. К этой иерархии мы применили новый метод получения первых интегралов ОДУ, основанный на факторизации гамильтоновых операторов при отображениях Миуры. Хотя такие первые интегралы ОДУ приводились ранее, принятый здесь обший 
подход ранее не описывался. Более точная формулировка этого метода будет представлена в другой работе. В другой работе будет приведен также обший анализ связи между преобразованиями Беклунда и отображениями Миуры для четвертой иерархии Пенлеве.

Мы также провели анализ особенностей второго члена четвертой иерархии Пенлеве и нашли, что алгоритм АРС дает сбой при применении к такому ОДУ. Это важно, потому что алгоритм АРС является стандартным тестом структуры особенностей, используемым как при тестировании уравнений на интегрируемость, так и в классификации Пенлеве. Мы представили решение задачи, даваемой этим уравнением. Полное изложение будет приведено в работе [11].

Благодарности. Данная работа частично поддержана со стороны DGICYT, контракты № РB98-0262 и № BFM2002-02609. Я благодарен организаторам NEEDS 2002, DGICYT в рамках контракта № PB98-0262, а также университету Саламанки за финансовую поддержку, позволившую мне принять участие в конференции.

\section{Список литературы}

[1] U. Muğan, F. Jrad. J. Phys. A. 1999. V. 32. P. 7933-7952; J. Nonlinear Math. Phys. 2002. V. 9. P. 282-310; C. M. Cosgrove. Stud. Appl. Math. 2000. V. 104. P. 1-65.

[2] M. J. Ablowitz, H. Segur. Phys. Rev. Lett. 1977. V. 38. P. 1103-1106.

[3] H. Airault. Stud. Appl. Math. 1979. V. 61. P. 31-53.

[4] P. R. Gordoa, A. Pickering. Europhys. Lett. 1999. V. 47. P. 21-24.

[5] P. R. Gordoa, A. Pickering. J. Math. Phys. 1999. V. 40. P. 5749-5786.

[6] P. R. Gordoa, A. Pickering. J. Phys. A. 2000. V. 33. P. 557-567.

[7] P.R. Gordoa, N. Joshi, A. Pickering. Publ. RIMS Kyoto Univ. 2001. V. 37. P. 327-347.

[8] Д. Леви, О. Рагниско, М. А. Родригес. ТМФ. 1992. Т. 93. С. 473-480.

[9] B. A. Kupershmidt. Commun. Math. Phys. 1985. V. 99. P. 51-73.

[10] M. J. Ablowitz, A. Ramani, H. Segur. J. Math. Phys. 1980. V. 21. P. 715-721.

[11] P. R. Gordoa, N. Joshi, A. Pickering. Publ. RIMS Kyoto Univ. 2003. V. 39. P. 435-449.

[12] A. Pickering. Testing Nonlinear Evolution Equations for Complete Integrability. Ph. D. thesis. University of Leeds, 1992.

[13] R. Conte, A. P. Fordy, A. Pickering. Phys. D. 1993. V. 69. P. 33-58. 